\title{
D'Aby Warburg à Éric Weil : le rêve d'une bibliothèque humaniste au 20e siècle
}

\section{Édouard Mehl}

\section{Q OpenEdition}

1 Journals

Édition électronique

URL : http://journals.openedition.org/rbnu/1382

DOI : 10.4000/rbnu.1382

ISSN : 2679-6104

\section{Éditeur}

Bibliothèque nationale et universitaire de Strasbourg

\section{Édition imprimée}

Date de publication : 1 novembre 2015

Pagination : 60-69

ISBN : 9782859230616

ISSN : 2109-2761

\section{Référence électronique}

Édouard Mehl, «D'Aby Warburg à Éric Weil : le rêve d'une bibliothèque humaniste au 20e siècle », La Revue de la BNU [En ligne], 12 | 2015, mis en ligne le 01 mars 2020, consulté le 12 décembre 2020. URL : http://journals.openedition.org/rbnu/1382 ; DOI : https://doi.org/10.4000/rbnu.1382

\section{(c) (i) (2)(2)}

La Revue de la BNU est mise à disposition selon les termes de la Licence Creative Commons Attribution - Pas d'Utilisation Commerciale - Partage dans les Mêmes Conditions 4.0 International. 


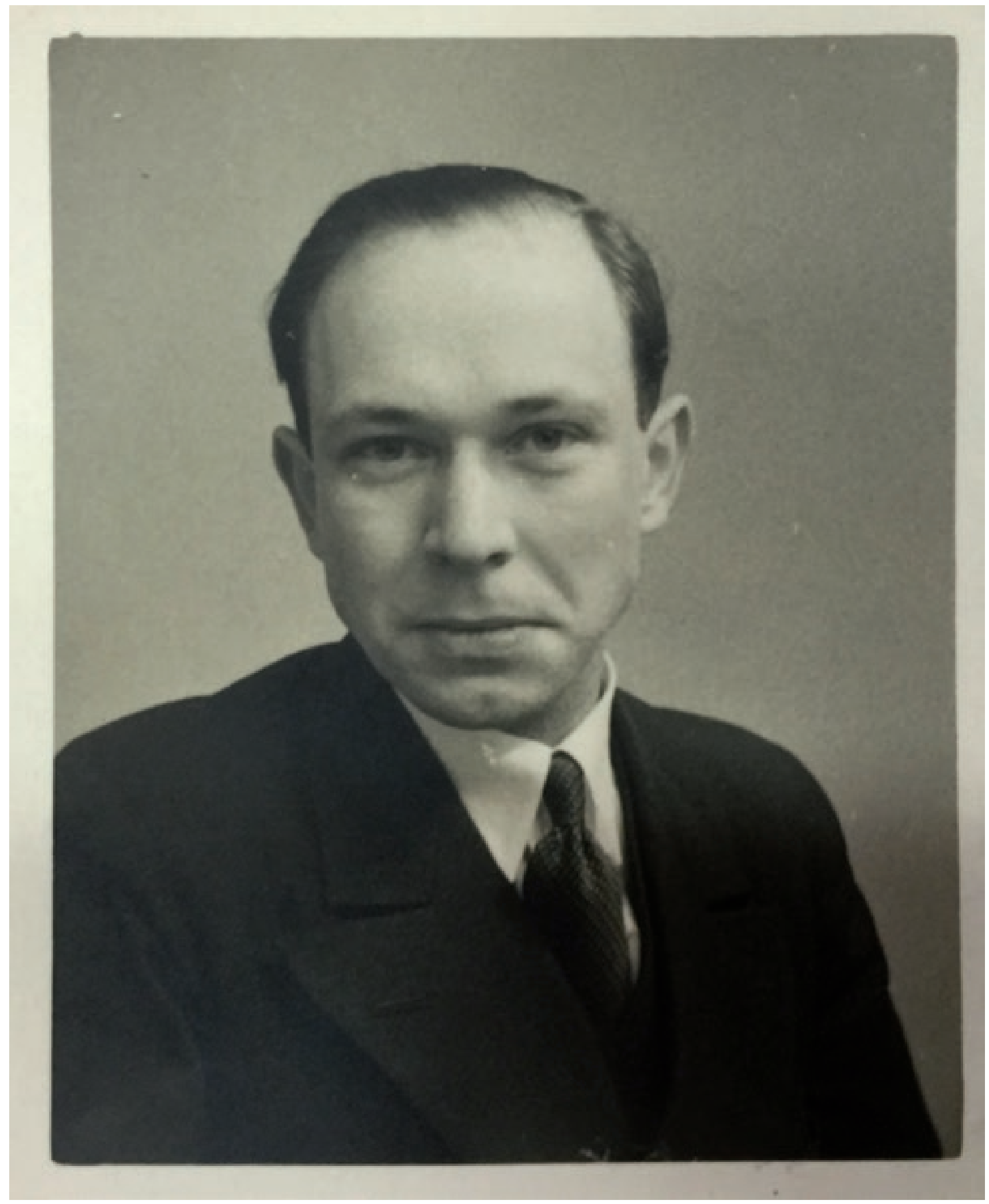




\section{D'Aby Warburg à Éric Weil : le rêve d'une bibliothèque humaniste au $20^{\mathrm{e}}$ siècle}

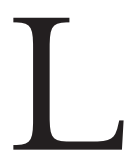

a vague d'autodafés qui a traversé toute l'Allemagne en mai 1933 compte parmi les épisodes les plus spectaculaires et les plus emblématiques des ravages que le nazisme a pu produire dans la culture. De Berlin à Nuremberg, de Königsberg à Francfort, et même jusqu'à Fribourg, nonobstant le rectorat du philosophe Martin Heidegger, des étudiants galvanisés, avec le soutien actif du corps professoral et de l'association des Volksbibliothekaren, ont brûlé par dizaines de milliers des ouvrages jugés contraires à l'esprit allemand - ou plus exactement, supposés émaner de "l'esprit nonallemand ". Quelques noms bien connus apparaissent dans un interminable inventaire : Stefan Zweig, Sigmund Freud, Karl Marx, mais aussi Henri Barbusse, Ernest Hemingway, ou - éloquent lapsus - tout ouvrage consacré à " Lautre " ${ }^{1}$. Entrée en vigueur en avril 1933, la non moins extravagante loi sur la fonction publique mettait d'office en retraite tout fonctionnaire non aryen. Dans le même temps, une génération entière d'aspirants aux distinctions académiques se chargeait de rendre l'enseignement de l'histoire, de la philosophie et des beaux-arts conforme aux exigences absurdes de l'idéologie national-socialiste. Les Reichsuniversitäten sont devenues, en l'espace de quelques mois seulement, des outils de propagande redoutablement efficaces en faveur de la pureté de la race, et du sacrifice de l'individu aux intérêts supérieurs du peuple.

Ces événements, pour incroyables qu'ils puissent aujourd'hui paraître, n'avaient pourtant rien d'imprévisible, et la plupart des intellectuels qui ont émigré cette année-là s'étaient déjà préparés à cette éventualité. Éric Weil (1904-1977), quittant l'Allemagne aussitôt après l'accession d'Hitler au pouvoir, n'a pas fait autre chose que son maître Ernst Cassirer, avec qui le jeune " Erich Weil " avait découvert l'astrologie, le platonisme de la Renaissance et les Lumières françaises ${ }^{2}$. Reprenons les choses de plus haut, en rappelant ici quelques éléments d'une histoire qui explique à la fois la trajectoire singulière d'Éric Weil et l'intérêt extraordinaire du fonds (quelques dix mille volumes) légué à l'Université de Lille 3, où Weil a enseigné de 1956 à 1968³

Éric Weil est aujourd'hui mieux connu comme le spécialiste de philosophie morale et politique qu'il est essentiellement devenu après-guerre, que comme l'historien de la Renaissance qu'il a d'abord été. Bien que nettement distincts, ces deux aspects de sa production scientifique sont pourtant indissociables, et ce dès les années d'études auprès de Cassirer ${ }^{4}$. Weil, en philosophe, s'intéresse davantage à comprendre l'émergence d'une nouvelle conscience de soi de l'homme à la Renaissance, qu'aux détails techniques de l'astrologie ou au passage progressif d'une physique des qualités à une science mathématique de la quantité, comme il pourra plus tard l'apprendre d'Alexandre Koyré dont, débarqué à Paris, il suit les leçons à l'École pratique des hautes études 5 . Du philosophe Cassirer, Weil aura surtout retenu l'idée générale que la magie et les savoirs pré-modernes présupposent un " monde " que la science moderne a proprement fait disparaître : " la science moderne ne connaît pas de cosmos, de système cohérent de la nature qui soit accessible à l'homme dans sa vérité, dans son être. A la place du cosmos, de la nature une, harmonieuse, belle, nous avons mis l'unité de la science, à la place des êtres liés les uns aux autres organiquement, nous avons mis les lois, les fonctions, les statistiques ${ }^{6}$.

Les recherches menées pendant les dernières décennies au Centre Éric Weil ont conduit à la publication des 


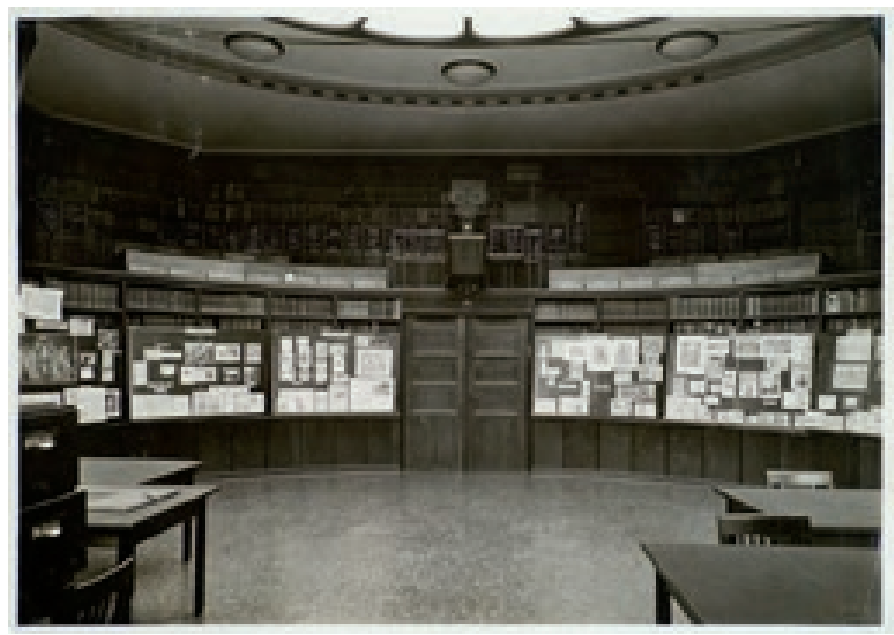

L'ellipse de la Warburg Haus (Hambourg)

deux travaux les plus importants de cette époque : La Philosophie de Pietro Pomponazzi, version française d'une thèse soutenue sous la direction d'Ernst Cassirer en 1928 et publiée dans les Archiv für Geschichte der Philosophie (1932) ; puis le mémoire sur Pic de la Mirandole et la critique de l'astrologie, reconnu en 1938 comme diplôme de l'École pratique (Ve section, Sciences religieuses), sous la direction de Koyré ${ }^{7}$ - avec pour rapporteurs le médiéviste Paul Vignaux et l'orientaliste Henry Corbin, excellent germaniste, qui fait paraître cette même année 1938 un recueil attendu de traductions d'Heidegger ${ }^{8}$.

Comme Max Lejbowicz et d'autres l'ont montré, ce travail sur Pic de la Mirandole, présenté pour l'obtention du diplôme français, peut être considéré comme le surgeon tardif d'un projet que l'émigration de 1933 devait faire avorter, celui d'une anthologie de textes astrologiques de la Renaissance. Ce travail devait tout son sens à un double patronage : celui, intellectuel, de Cassirer - auteur d'une publication décisive pour l'orientation initiale d'Erich Weil : Individuum und Kosmos in der Philosophie der Renaissance (1927) ; et celui, institutionnel et professionnel, du tout récent Institut Warburg de Hambourg, dans le cadre duquel Cassirer avait lui-même inscrit son propre travail ${ }^{9}$. Il n'est donc pas exagéré de considérer que c'est une bibliothèque privée, exceptionnelle et unique en son genre, qui a décidé de l'orientation d'Éric Weil - et qui a connu un destin analogue, puisque les fonds (65 ooo volumes, 25000 photographies) et le personnel scientifique de l'Institut ont été transférés à Londres en $1933^{10}$.
Abraham M. (Aby) Warburg (1866-1929) est l'aîné d'une famille de sept enfants. Une légende familiale, qui n'a aucune raison d'être fausse, dit que très tôt, en 1877, Aby cède son droit d'aînesse à son frère cadet, Max (et partant abandonne la succession de son père Moritz à la direction d'une banque qui allait devenir une des toutes premières institutions financières d'Allemagne), en échange de la promesse de se faire acheter tous les livres qu'il souhaite ${ }^{11}$. La promesse fut tenue, et même bien au-delà. Après des études d'histoire de l'art qui l'ont notamment conduit à fréquenter la toute nouvelle Université de Strasbourg, où il se familiarise avec l'histoire de la Renaissance italienne auprès d'Hubert Janitschek, dans des locaux eux-mêmes construits à l'imitation de l'architecte italien Sanmicheli, la colossale fortune familiale permit non seulement l'acquisition du fonds, mais aussi la construction d'un bâtiment adapté à recevoir des collections si variées que leur classement constitue en lui-même un problème et un défi à l'intelligence ${ }^{12}$. Warburg ne voulait pas entendre parler des systèmes de classement thématiques, alphabétiques ou chronologiques en vigueur dans la plupart des bibliothèques publiques. Une logique toute différente préside ici, celle du " bon voisinage " entre les ouvrages, "logique " qui s'impose si l'on admet, comme Warburg semble en avoir été convaincu, que le livre dont un chercheur a réellement besoin n'est pas celui qu'il cherche, mais son voisin. Aussi le bon ordre ne peut-il précéder la recherche et devrait-il, en principe, en découler. Pour parler le langage des géomètres, c'est un ordre analytique plutôt qu'un ordre synthétique, à 


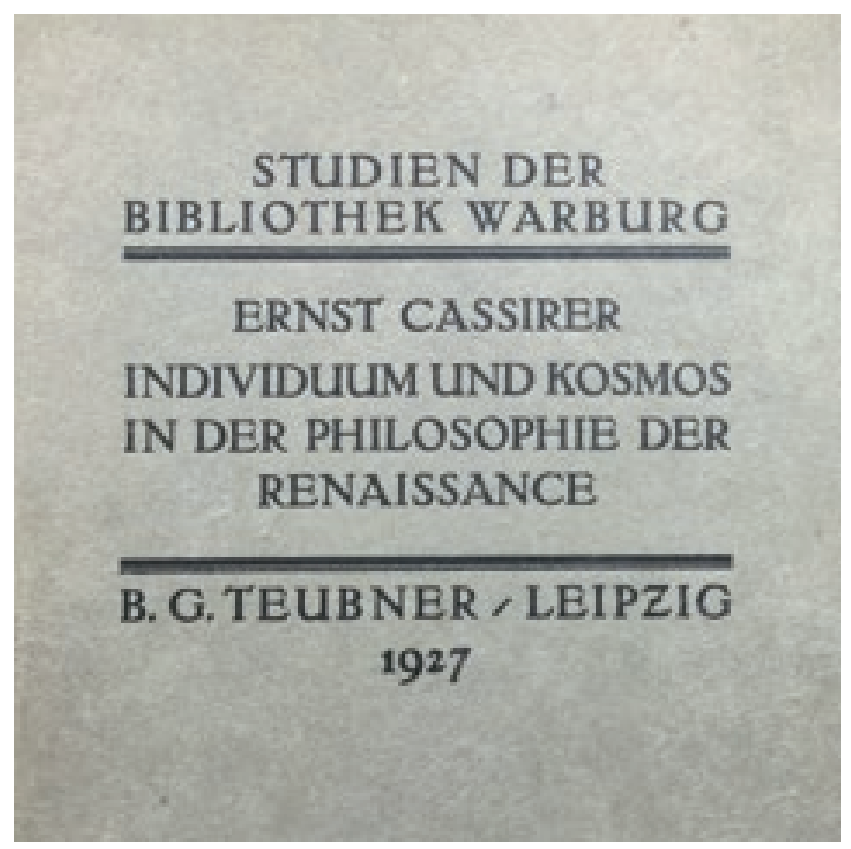

E. Cassirer, Individuum und Kosmos in der Philosophie der Renaissance (1927), publications de la Bibliothek Warburg, exemplaire personnel d’É. Weil (coll. Institut Éric Weil).

\section{A. W A R B U R G ZUM 6o.GEBURTSTAG A M I3. J U N I 926}

La dédicace de Individuum und Kosmos à Aby Warburg (coll. Institut Éric Weil).

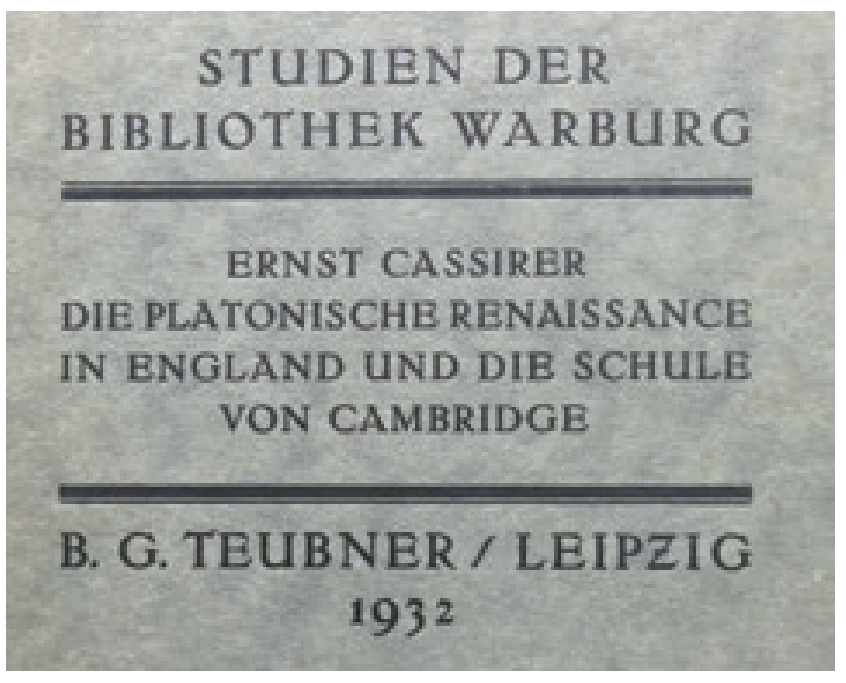

E. Cassirer, Die Platonische Renaissance in England und die Schule von Cambridge (1932); publications de la Bibliothek Warburg, exemplaire personnel d’É. Weil (coll. Institut Éric Weil).

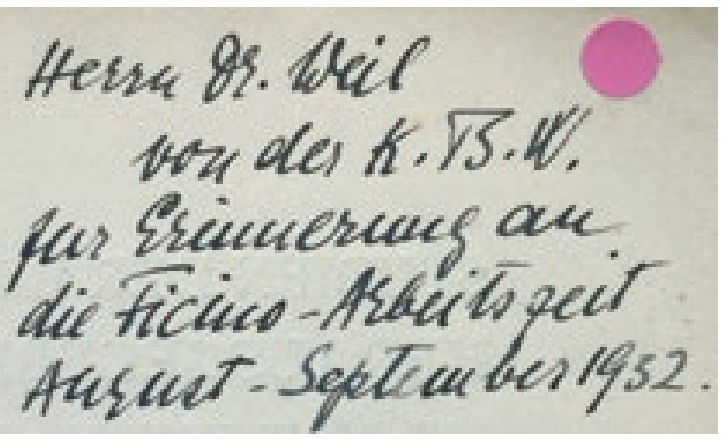

Dédicace manuscrite de l'ouvrage de Cassirer à Éric Weil [« Don de la Bibliothèque Warburg au Dr. Weil, en souvenir du séjour de travail sur Ficin, août-septembre 1932 "] (autographe vraisemblablement de la main de Fritz Saxl, bibliothécaire ; coll. Institut Éric Weil). 


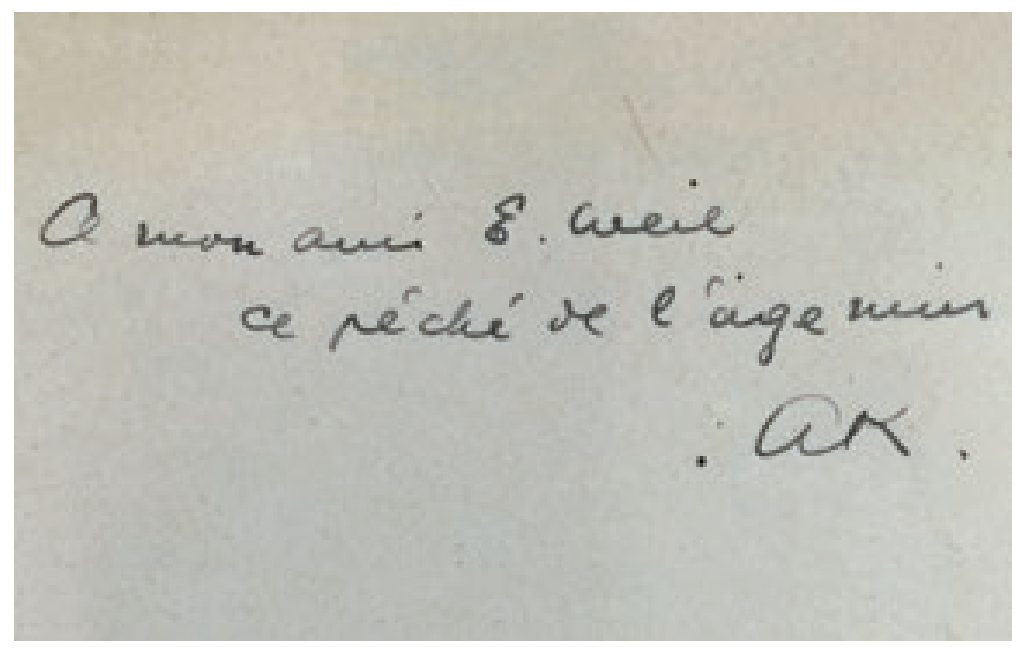

Dédicace d'Alexandre Koyré, La Philosophie de Jacob Boehme (1929):

"A mon ami É. Weil, ce péché de l'âge mûr " (coll. Institut Éric Weil).

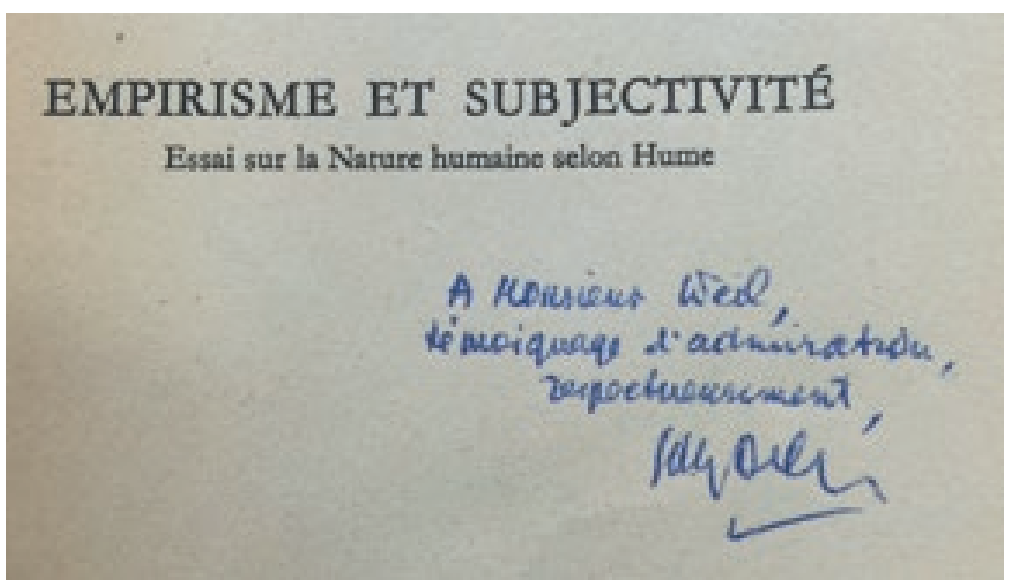

Dédicace de Gilles Deleuze,

Empirisme et subjectivité (1953; coll. Institut Éric Weil).

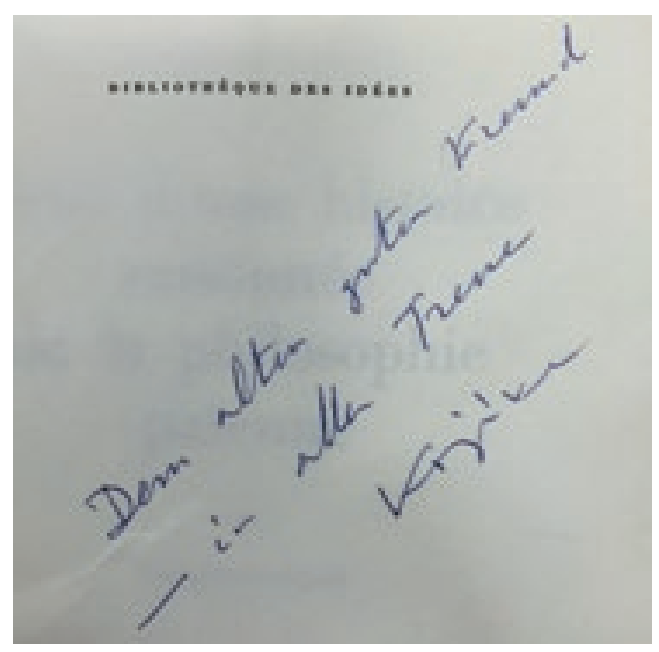

Dédicace d'Alexandre Kojève, Essai d'une histoire raisonnée de la philosophie païenne (1968) [ "Au bon vieil ami. Fidèlement, Kojève "] (coll. Institut Éric Weil).

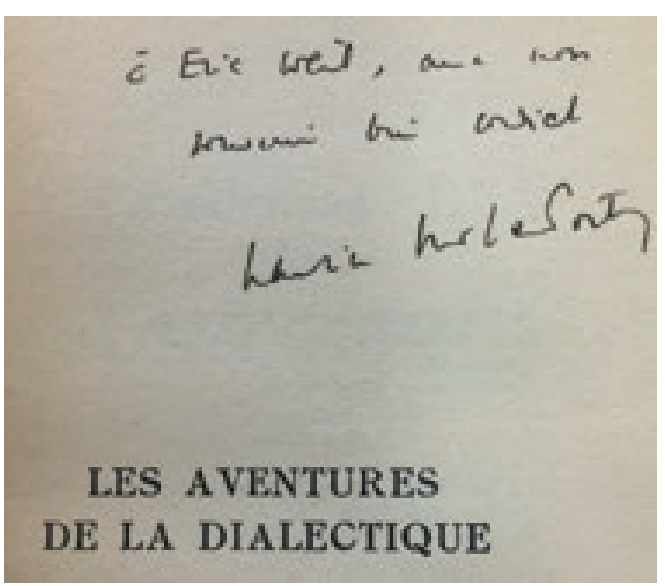

Dédicace de Maurice Merleau-Ponty, Les Aventures de la dialectique (1955; coll. Institut Éric Weil). 


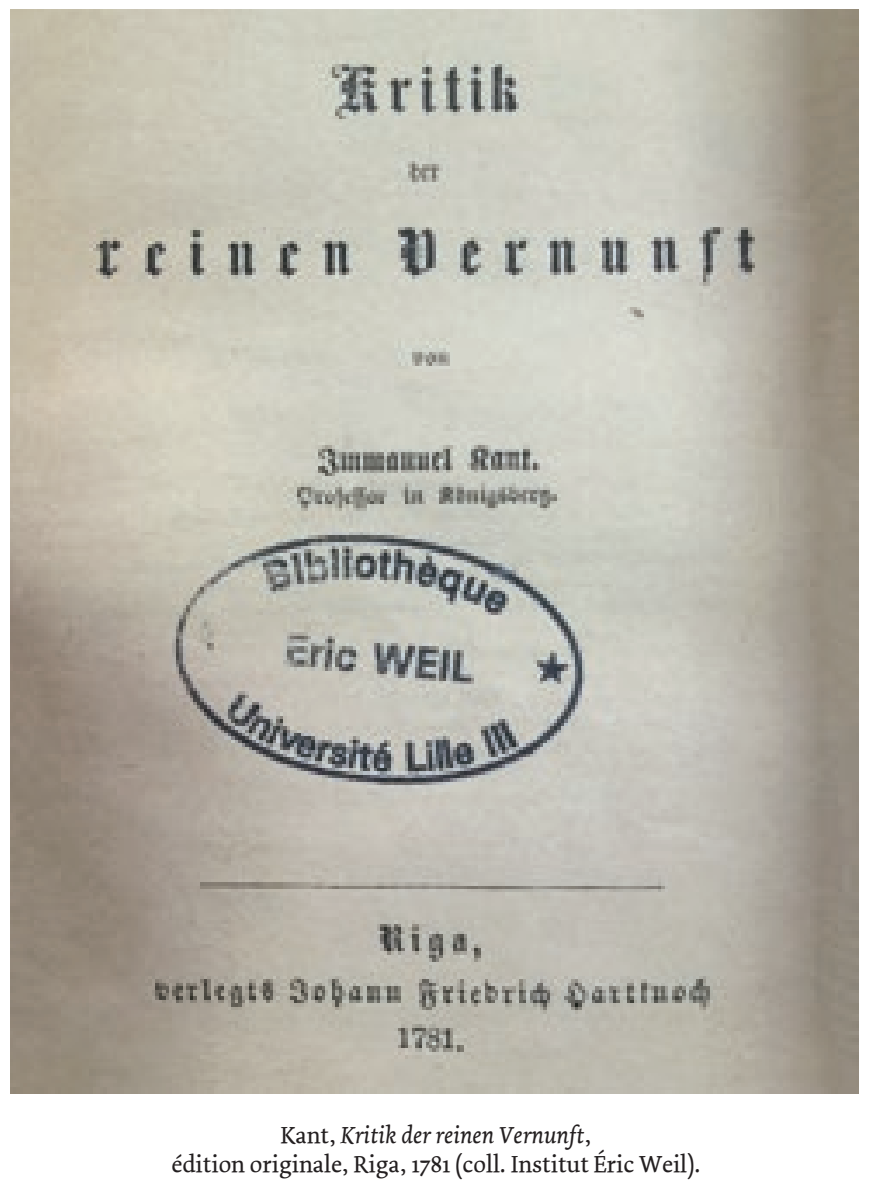

l'usage exclusif des chercheurs, et moins à vrai dire fait à que par l'usage des chercheurs, comme si le pas d'un marcheur dessinait le chemin qui s'ouvre devant lui.

Cette originalité se redouble d'une question vraiment abyssale, qui avait déjà frappé Cassirer dès le début des années 1920 : le problème de méthode découle en fait d'un problème d'objet - ce qu'indique assez bien la déclaration de Cassirer, ouvrant la série des conférences du Centre Warburg : "J'ai éprouvé de la manière la plus impérieuse que nous n'avons pas ici affaire à une simple collection de livres, mais bien davantage à une collection de problèmes. Plus encore que le domaine thématique de cette bibliothèque, c'est le principe de sa construction qui a suscité en moi cette impression : car ici l'histoire de l'art, l'histoire des mythes et des religions, l'histoire des langues et des cultures n'étaient manifestement pas simplement juxtaposées : elles étaient rapportées les unes aux autres et toutes ensemble à un idéal point central ${ }^{13}$. De fait, même si ce qui est ici indiqué ne suffit pas à faire toute la lumière sur un " principe de construction " qui garde son secret, l'analogie de cet espace de travail organisé autour d'un idéal " point central " avec le cosmos de la Renaissance dont l'homme occupe encore supposément le " centre " est trop évidente pour avoir pu échapper à Cassirer.
Tout se passe donc comme si la Kulturwissenschaftliche Bibliothek Warburg refusait a priori la disciplinarisation, et comme si l'agencement subtil et aléatoire de ce chaos était l'unique moyen d'atteindre une compréhension non objectivante de l'homme moderne. Au fond, l'intérêt de Warburg pour la Renaissance italienne n'est que la face émergée d'une recherche inquiète et d'une inquisition sans fin sur un objet indéfinissable et sans essence assignable : l'homme, ce monstre que le grand pourfendeur du déterminisme astrologique, Pic de la Mirandole, présentait comme un être indéfinissable, tenant moins du créateur l'être au sens d'une essence propre, que le pouvoir de se la donner à lui-même. Aussi faut-il prendre au sérieux et au pied de la lettre la formule que donnait Warburg lui-même du projet partagé avec Cassirer de produire une "science culturelle générale comme doctrine de l'homme en mouvement " (allgemeine Kulturwissenschaft als Lehre vom bewegtem Menschen) ${ }^{14}$, ce " mouvement " n'étant autre que le passage d'une forme à une autre, reflet fugitif d'une liberté qui traverse l'histoire et ne s'y repose jamais.

Ces quelques éléments permettent de comprendre que la bibliothèque personnelle léguée par Éric Weil à l'Université de Lille 3 porte un héritage philosophique considérable : celui d'un témoin exceptionnel du $20^{\mathrm{e}}$ siècle, 


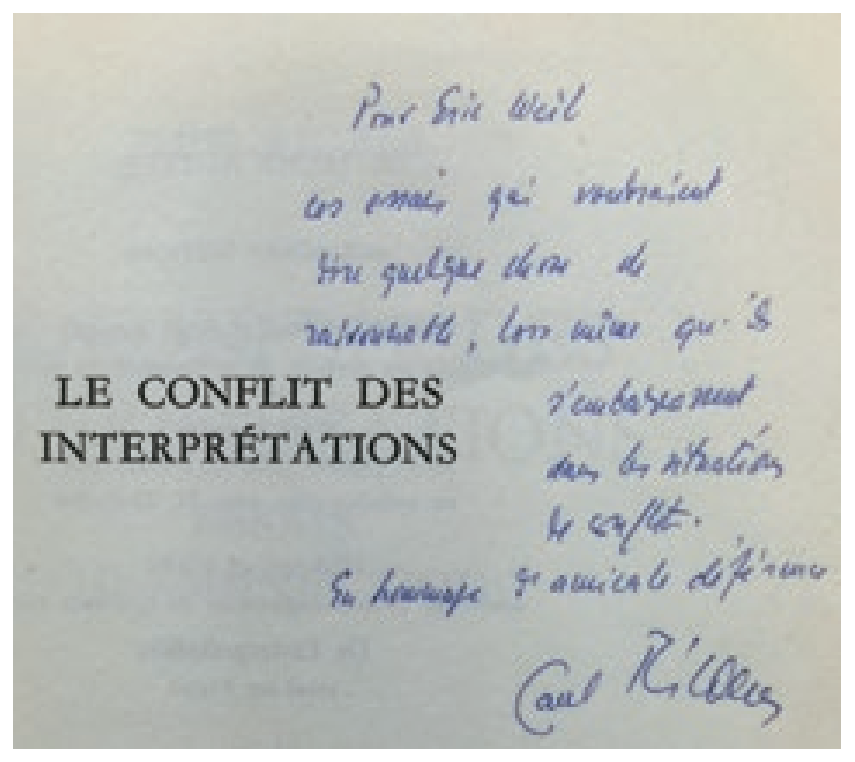

Dédicace de Paul Ricoeur, Le Conflit des interprétations. Essais d'herméneutique (1969; coll. Institut Éric Weil).

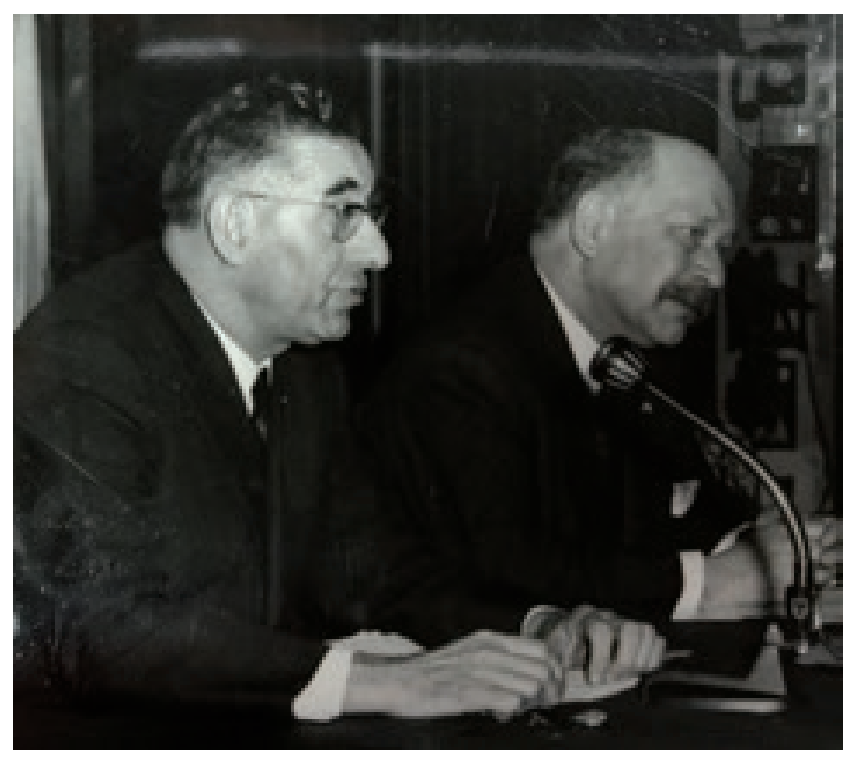

Éric Weil et Paul Ricoeur, février 1967 (coll. Institut Éric Weil). qui a fréquenté aussi bien Cassirer que Georges Bataille (avec qui il anime, nonobstant leurs évidentes divergences de vue, la revue Critique dans les années de l'immédiat après-guerre) $)^{15}$, et dont les centres d'intérêt sont très loin de se restreindre à l'histoire de la philosophie entendue comme discipline académique. Aussi le fonds, d'une très grande richesse, présente-t-il quelques traces de l'histoire que nous avons ici évoquée : comme pour la Kulturwissenschaftliche Bibliothek Warburg, la diversité a imposé une méthode de cotation spécifique ${ }^{16}$. Les ouvrages strictement philosophiques (parmi lesquels une édition originale de la Critique de la raison pure (1781), la collection complète des EFuvres de Jean-Jacques Rousseau (Genève, 1782) ou un exemplaire annoté de Être et Temps) voisinent avec quelques illustres témoignages de l'intérêt de Weil pour l'humanisme italien (Pétrarque, Politien, Ficin...). Une partie du fonds provenant de la famille de son épouse Anne Mendelsohn, amie d'enfance d'Hannah Arendt, et de sa belle-sœur Catherine, on ne peut pas présupposer que tous les ouvrages du fonds aient été la propriété personnelle d'Éric Weil ni ses instruments de travail. Bien que la bibliothèque constitue par ellemême un instrument d'un intérêt immense pour étudier la pensée du philosophe, la plus élémentaire prudence méthodologique impose cependant de rappeler ici ce que l'on a pu dire, par exemple, à propos de la bibliothèque de Spinoza : ce n'est pas parce qu'un livre ne s'y trouve pas qu'il ne l'a pas lu... et ce n'est pas non plus parce qu'il s'y trouve qu'il l'a lu !

Ajoutons enfin, pour conclure cette présentation succincte, quelques éléments sur les conditions légales et institutionnelles qui ont rendu possible l'accès public à la Bibliothèque Éric Weil. Dans les années 1980, le Centre Éric Weil avait le statut universitaire d’Équipe d'accueil, dotée d'une revue destinée à en diffuser les travaux (les Cahiers Éric Weil, premier numéro publié en 1987). Regroupé en 2006 au sein de l'UMR 8163 du CNRS (Savoirs, Textes, Langage), le Centre Weil a cessé d'exister en tant que tel. Créé en 2011, l'« Institut Éric Weil » a pris le relais des activités du Centre. Il favorise l'étude et la diffusion de l'œuvre philosophique d'Éric Weil, mais aussi, selon les statuts du Centre, "la réflexion et le dialogue, en liaison avec la formation des étudiants, sur les problèmes fondamentaux qu'elle posait : problèmes de philosophie première (de la compréhension et du sens, de la possibilité du dialogue, de la dialectique de la raison et de la violence, etc.), de philosophie de la praxis (morale, politique, éducation, histoire), d'histoire de la philosophie (héritage de Hegel et de Kant, de Heidegger et de Marx, d'Aristote...) ". L'Institut est doté d'un conseil de gestion où sont notamment intégrés Jean Quillien et Gilbert Kirscher, tous deux anciens étudiants de Weil et exécuteurs testamentaires du legs WeilMendelsohn. Outre la gestion de la bibliothèque - dont 

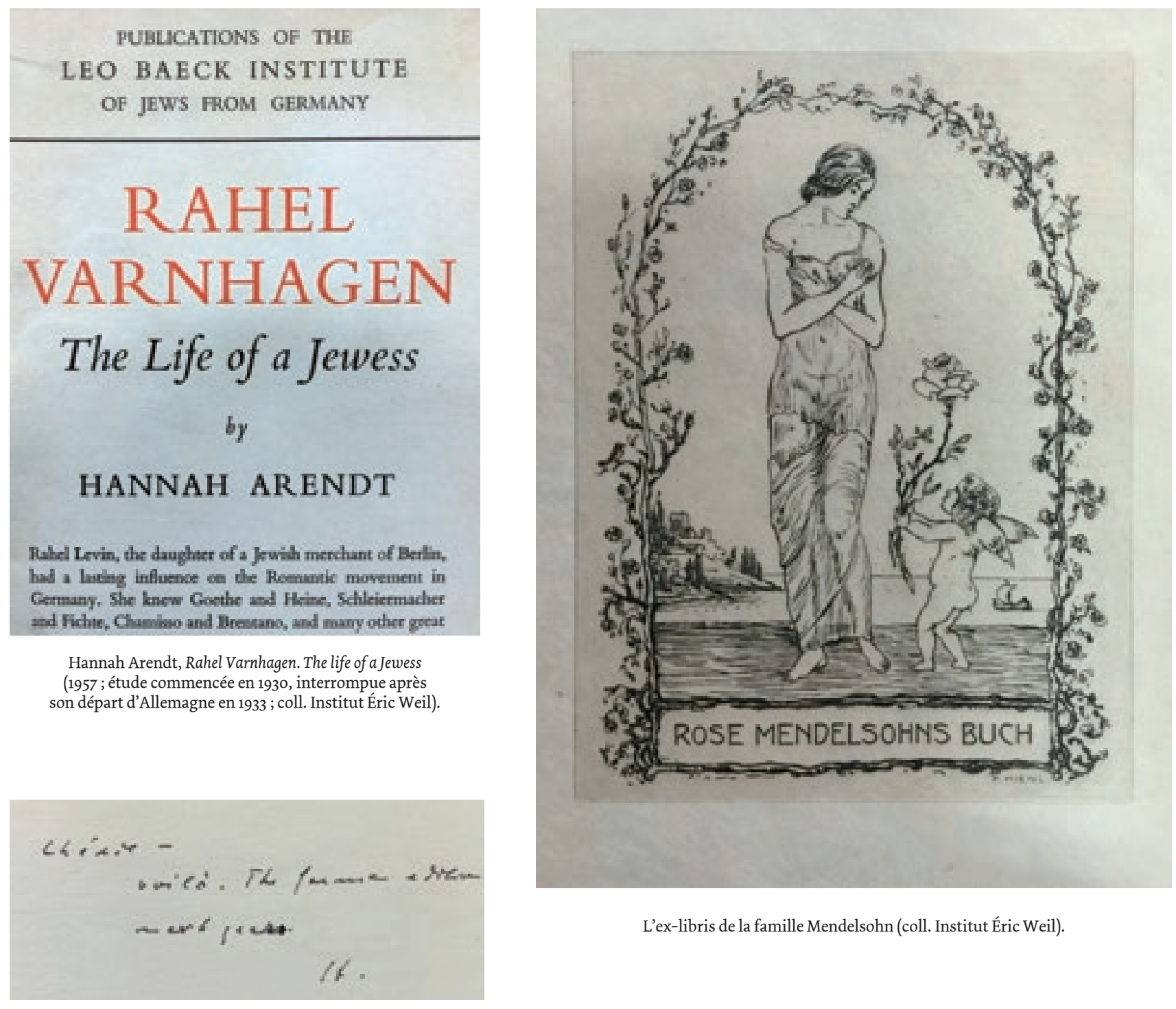

L’ex-libris de la famille Mendelsohn (coll. Institut Éric Weil).

Dédicace d'H. Arendt à son amie Anne Weil-Mendelsohn : "Chérie - voilà. The former edition next year[s]" (coll. Institut Éric Weil).

le fonds, initialement constitué d'une dizaine de milliers d'ouvrages, s'enrichit par l'acquisition systématique de la littérature consacrée au philosophe, ainsi que par la numérisation progressive des archives, comprenant une part de la correspondance et des manuscrits inédits ${ }^{17}$-, l'Institut a vocation à favoriser l'accueil de chercheurs, le développement de partenariats internationaux et l'animation de la recherche autour d'Éric Weil. Le succès de cette entreprise et la fécondité actuelle des études weiliennes ne dépendent donc pas seulement du don luimême : ils dépendent aussi du fait que la communauté universitaire a su créer les conditions propices à sa réception et qu'elle a la volonté de faire acte de mémoire en poursuivant une œuvre qui reflète admirablement toute l'histoire, douloureuse, violente, tragique, $\mathrm{du} 2 \mathrm{O}^{\mathrm{e}}$ siècle.

\section{Édouard Mehl}




\section{Notes}

1 - Sic, pour Henri de Toulouse-Lautrec. Voir pour une réaction contemporaine à ce sinistre épisode : Stefan Zweig, Le monde d'hier. Souvenirs d'un européen, trad. Jean-Paul Zimmermann, Paris, Les Belles lettres, 2013.

2 - Mais bien d'autres noms devraient s'ajouter à ceux-là : Paul Tillich, Karl Löwith, Leo Strauss, Hannah Arendt, pour ne citer que quelquesuns des plus célèbres. Dans l'entourage immédiat de Weil, il faut encore citer le philosophe Max Dessoir (Max Dessauer, 1867-1947), dont Weil est le secrétaire particulier jusqu'à ce que Dessoir soit lui-même privé de sa chaire en 1933, en raison de ses origines juives.

3 - Créé en 2011, l'Institut Éric Weil, actuellement dirigé par le professeur Patrice Canivez, a notamment vocation à gérer la bibliothèque Éric Weil hébergée au sein de la Bibliothèque universitaire de l'Université de Lille 3.

4 - Unité bien soulignée par Francis Guibal, Le courage de la raison. La philosophie pratique d’Éric Weil, Paris, Les Éditions du Félin, 2009, ch. I ("Les choix d'une vie»).

5 - Koyré a donné la première traduction française du De revolutionibus orbium coelestium (Paris, F. Alcan) en 1934. Il tient deux séminaires, confiés à Alexandre Kojève et Henry Corbin au cours de l'année 1937-1938, pendant laquelle Koyré occupe une chaire à l'Université du Caire. Corbin donne, pour cette année 1937-1938, un cycle de conférences sur " L’inspiration luthérienne de J. G. Hamann ", dans la droite ligne des études que Koyré consacre, dans le cadre de la chaire d'histoire des idées religieuses, aux mystiques et spirituels du temps de la Réforme (Sebastian Franck, Caspar von Schwenckfeld, Paracelse, Valentin Weigel), tous supposés plus luthériens que Luther lui-même - études réunies dans Mystiques, spirituels, alchimistes du XVI siècle allemand, Armand Colin, 1955, puis Gallimard, 1971. Dans le même temps, Kojève étudie les deux derniers chapitres de la Phénoménologie de l'esprit (La religion, Le savoir absolu).

6 - É. Weil, texte d'une conférence inédite, «Science et magie » $(1949$, f. 4), d'après le manuscrit numérisé par l'Institut Éric Weil : http:/|eric-weil. recherche.univ-lillez.fr. La citation que nous retranscrivons ici résume au fond assez bien ce qui pourrait être l'idée directrice de Substance et fonction d'E. Cassirer (Substanzbegriff und Funktionsbegriff, Hamburg, 1910), dont Weil ne retient ici que l'aspect purement négatif : la révolution copernicienne comme liquidation et destruction du cosmos. Une idée quà vrai dire le second maître de Weil, Alexandre Koyré, partage également (voir par exemple l'avant-propos de Du monde clos à l'univers infini : Koyré veut dépeindre l'émergence de la Weltbild moderne comme « la destruction du cosmos " et l'aboutissement d'un processus par lequel l'homme a " perdu le monde même qui formait le cadre de son existence ", processus aboutissant catastrophiquement à la " dévalorisation complète de l'être et au divorce total entre le monde des valeurs et le monde des faits " (Gallimard, TEL, 1973, p. 11-12).

7 - Éric Weil, La Philosophie de Pietro Pomponazzi ; Pic de la Mirandole et la critique de l'astrologie, Paris, Vrin, 1986. Nous devons essentiellement la connaissance et la réédition de ces travaux d'avant-guerre aux recherches de Max Lejbowicz, « Éric Weil et l'histoire de l'astrologie. Éléments d'un itinéraire ", in Cahiers Éric Weil, vol. 1. Textes réunis par J. Quillien, Lille, Presses universitaires du Septentrion, p. 93-122. Notons que la Cinquième section de l'EPHE, présidée par Marcel Mauss, n’a diplômé que quatre étudiants en 1937-1938, dont Michel Leiris, pour un mémoire sur " Le langage secret des Dogons de Sanga ".

8 - Sur Henry Corbin et l'introduction de la pensée d'Heidegger en France, voir la nouvelle étude de Sylvain Camilleri et Daniel Proulx, Bulletin Heidegger 4 [Bhdg] (2014), Liminaire 1 : " Martin Heidegger - Henry Corbin (Lettres et documents, 1930-1941) ", p. 4-63. Cette excellente étude évoque à juste titre le Congrès Descartes de 1937 (année du tricentenaire du Discours de la méthode) où Henry Corbin a officieusement représenté Heidegger, absent pour des raisons qui ont fait couler beaucoup d'encre. Rappelons seulement ici que la délégation allemande à ce congrès était conduite par le nazi Hans Heyse, recteur de Königsberg entre 1933 et 1935, dont la lecture de Descartes renvoie explicitement à celle d'Heidegger (Idee und Existenz, Hamburg, 1935, p. 181). Étant donné la composition de la délégation allemande, Heidegger ne pouvait en aucun cas participer à ce congrès sans que cela ne fût immédiatement interprété comme le signe d'un engagement non équivoque en faveur du nazisme. Mais l'insistance avec laquelle Heidegger demande à Corbin, au moment précis du congrès, de récuser toute lecture " existentialiste " de Être et Temps " pour se garder de toute mésinterprétation anthropologique " (Martin Heidegger an Henry Corbin, 15 mars 1937, éd. S. Camilleri et D. Proulx, Bhdg 4, p. 27-30) peut aussi se comprendre comme un désaveu implicite, mais formel de la " philosophie " de Heyse - caricature grossière et grotesque de la sienne.

9 - Cassirer a lui-même dédié son ouvrage à Aby Warburg, soulignant l'importance pour ses propres recherches d'une bibliothèque qui n'avait pas seulement vocation à classer les productions scientifiques selon l'ordre des matières, mais en fonction de leur idéale convergence vers / à partir d'un point central.

10-Voir à ce sujet l'article de Raphaële Mouren p. 51

11 - Sur l'histoire de la dynastie et cette anecdote, voir J. Attali, Sir Siegmund Warburg (1902-1982). Un homme d'influence, Paris, Fayard, 1985, p. 68-69; p. 91.

12-J'emprunte une partie de ce qui suit à Maud Hagelstein, " Mémoire et Denkraum. Réflexions épistémologiques sur la Kulturwissenschaftliche Bibliothek Warburg ", in Conserveries mémorielles, \#5 | 2008, mis en ligne le $1^{\text {er }}$ octobre 2008, consulté le 30 juillet 2015 (http://cm.revues.org/104).

13 - E. Cassirer, " Der Begriff der symbolischen Form im Aufbau der Geisteswissenschaften ", in Vorträge der Bibliothek Warburg. Vorträge 1921/22. Leipzig et Berlin : Teubner, 1923, p. 11-39; texte cité par J. Faehndrich dans Ernst Cassirer und Aby Warburg. Ein Literaturbericht (Magisterarbeit am Institut für Kulturwissenschaften der Universität Leipzig, 2000 ; notre traduction).

14-Selon une lettre de Warburg à Cassirer du 15 avril 1924, citée par Muriel van Vliet, " Rituel et mythe chez Warburg, Cassirer et LéviStrauss ", in Appareil [articles mis en ligne le 7 octobre 2014], consulté le 31 juillet 2015 (http://appareil.revues.org/2074).

15- Signalons l'édition récente de la correspondance par Sylvie Patron : Georges Bataille - Éric Weil. A en-tête de Critique. Correspondance 1946-1951, Lignes, 2014

16-http://eric-weil.biblio.univ-lille3.fr/Presentation.html

17- Précisons ici que l'Institut Éric Weil n'a pas vocation à conserver ni même à recenser l'intégralité de la correspondance, dispersée ici et là : l'IMEC (Institut Mémoires de l'édition contemporaine) conserve la plus grosse partie de la correspondance consacrée à la revue Critique; le CAPHES (Centre d'archives en philosophie, histoire et édition des sciences, qui a pour mission de valoriser les fonds d'archives hébergés à l'ENS, comme entre autres le fonds Georges Canguilhem (http:/|caphes.ens.fr/ IMG/file/inventaire_G.Canguilhem.pdf), a inventorié également plusieurs lettres d'É. Weil jointes à la correspondance passive de Jean Hyppolite. Parmi les manuscrits inédits présentés par l'IEW, on notera plusieurs textes consacrés à l'université et à la vie étudiante, comme celui que Weil a consacré à la réforme de l'université en 1968, qui n'a pas rien perdu de son intérêt ni de sa pertinence (cf. illustration ci-contre). 
b) - Cole copondant n'eat probeblement pes 1'essentiel. Il sesble inoomparablement plus important de joindre th l'instruotion 1'6dueation de $1^{\prime}$ homae.

Ce qui a été proposé sous a) y sera certaineaent utile fact que puiseque l'ouverture de perspetive, nouvelles et inconnues conetitue par elle-n8me un enriohiesenent. M6anmoins, 11 faudra pout-8tre penser, préoisénent à ce niveau, à une réorientation fondamentale de l'esprit univereitaire.

bu fond des mouvements violents de la jeunesse pré-6́tudiante et étudiante, mouvexents qui ont touohé toue les Btate, toutes les Socíttés modernex, se renoontre une insatisfaction qui n'arrive pas a se penser. La confusion des "idées" proclamées est 6vidente et rien ne serait plus facile que de les rófuter. Mais oe que oe mouvenent exprime nal $\mathrm{n}^{\prime}$ eh est pas pour autant sane justification.

On proteste couranment contre la socít6 de consomation. Il eet olair que pereonne $\mathrm{n}^{\prime a}$ envie de retourner dans les for Xa1s le sens de la protestation est peut-être autre que n'arrivent i. penser leo protentataires. Ce contre quoi ils se dressent; pourrait être facilement un monde de la richesse ot de $1 \mathrm{a}$ consomation parement astérielleg dans lequel tout ce qui a un sens

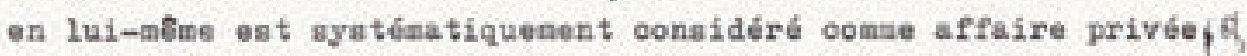
par conebquent, eane importance "r6́elle".

La poteie, l'art, la nature, 1a pensée "desintéress6e", o'est-d-dire sans omplol posoible dano le proceseus de produotion matérielle, sont respectéts, voire prônéłs, mais ne sont pes pris au sérieux dans le sens ol le travail productif ou organisationnel l'est.

$$
\ldots / \ldots
$$

